\title{
Neutrinos in Extra Dimensions and the Anomalous Magnetic Moments of Leptons
}

\author{
G. C. McLaughlin] and J. N. Ng] \\ TRIUMF, 4004 Wesbrook Mall, Vancouver, B.C., Canada V6T 2A3
}

\begin{abstract}
The use of extra dimensional scenarios as models for neutrino mass affects many low energy observables. We consider the implications of virtual bulk neutrinos in precision experiments of the anomalous magnetic moments of the muon and the electron. We consider neutrino models in factorizable geometry of the type $M_{4} \times T$ as well as the sliced $A d S_{5}$ non-factorizable geometry. In both geometries we find finite contributions to $g-2$ after summing over the KK excitations of the bulk neutrinos. In the case of Randall-Sundrum geometry, we find that the muon experiment is approaching the precision necessary to probe these models.
\end{abstract}

\footnotetext{
${ }^{1}$ email: gail@triumf.ca

${ }^{2}$ email: misery@triumf.ca
} 
The existence of extra dimensions is a common feature of many extensions of the Standard Model (SM). In particular it is well known that string theory can be formulated consistently in 10 or 11 dimensions. Usually the higher dimensional geometry is taken to be $M_{4} \times T$ where $M_{4}$ is Minkowski space and $T$ denotes the compactified space of the extra dimensions. The extra dimensions are usually taken to be spatial and the geometry of $T$ is assumed to consist of spheres or tori with small radii. Therefore, the extra dimensions avoid detection in current experiments. Recently, it has been suggested that extra dimensions can be used to explain the hierarchy between the weak and the Planck scale. There are two distinct scenarios which implement this idea. In one case the extra dimensions are taken to be large [1, 2] and the apparent hierarchy is due to the large volume of the extra dimensions. Specifically, the fundamental scale $M_{*}$ in $4+n$ dimensions where $n$ denotes the number of extra dimensions is related to the $4 \mathrm{D}$ Planck scale $M_{P l}$ via the relation

$$
M_{P l}^{2}=M_{*}^{n+2} V_{n}
$$

and $V_{n}$ is the volume of the compactified extra dimensions. For simplicity we shall take the geometry of this compactification to be a $n$-dimensional torus with equal radii $R$ and thus $V_{n}=(2 \pi R)^{n}$. If $R$ is of the order of $1 \mathrm{~mm}$ then $M_{*}$ can be taken to be as low as $\mathrm{TeV}$ for $n \geq 2$. In order to accommodate the success of the SM and the non-observation of a tower of Kaluza-Klein excitations of SM particles, one can simply confine all fields charged under the SM gauge group to a 3D hypersurface. This can be achieved by D-branes [3] of string theory.

A second scenario pioneered by Randall and Sundrum (RS) [4] solves the hierarchy problem by introducing a warp factor to the $4 \mathrm{D}$ metric. This construction makes use of one extra dimension which is parametrized by the coordinate $z=r_{c} \phi$ and the points $\left(x^{\mu}, \phi\right)$ and $\left(x^{\mu},-\phi\right)$ are identified. Here, $x^{\mu}$ denotes the usual 4D Minkowski space coordinates. The metric is given by

$$
d s^{2}=e^{-2 k r_{c}|\phi|} \eta_{\mu \nu} d x^{\mu} d x^{\nu}-r_{c}^{2} d \phi
$$

where the metric $\eta_{\mu \nu}$ has the signature (+ - - -). The full five dimensional metric will be later referred to as $G_{A B}$. The parameter $k$ is a measure of the curvature of the compactified dimension. For consistency one would expect $k \lesssim M_{5}$ where $M_{5}$ is the fundamental 5D scale. The construction also uses two branes, one located at $\phi=0$ which is referred to as the hidden brane and another at $\phi=\pi$ where the SM particles are localized. We shall refer to the latter as the visible brane. The effective Planck scale $M_{P l}$ as seen by observers confined to the visible brane is

$$
M_{P l}^{2}=\frac{M_{5}^{3}}{k}\left(1-e^{-2 k r_{c} \pi}\right) .
$$

Unlike the first scenario the radius $r_{c}$ is not large and the hierarchy between the weak and the Planck scale is solved by taking $k r_{c} \approx 12$. 
While the use of extra dimensions to solve the hierarchy problem is very intriguing it also brings with it many new challenges. In particular, in the area of neutrino physics it abjures the generation of small neutrino masses via the seesaw mechanism. This is because large mass scales are absent from the theory and the only operating scale in the visible brane is the $\mathrm{TeV}$ mass scale. Instead, in both scenarios neutrino masses are generated by allowing the SM singlet fermion, which is usually referred to as the righthanded neutrino, to be a bulk field just like the graviton. Bulk neutrino are discussed in the context of the factorizable geometry by Arkani-Hamed, Dimopoulos, and Dvali (ADD) [5]. The smallness of the neutrino mass is due to the scaling of the Yukawa coupling by the volume of the large extra dimensions. Solutions to the atmospheric neutrino and solar neutrino anomalies are discussed in [6, 0]. Further phenomenological studies can be found in 8$]$.

The active neutrino receives a small mass in a different way in the RS model. The bulk neutrino has a zero mode with a very small wave function at the visible brane [9]. After spontaneous symmetry breaking, with both the Higgs field and the lepton doublet residing on the visible brane, a light neutrino of mass in the range of $10^{-2}$ to $1 \mathrm{eV}$ range is generated (for details see [9]). This can be generalized to Higgs fields in the bulk [10]. All of these models for producing neutrino mass in an extra dimensional scenario represent the simplest constructions and contain no new additional particles and have no new gauge interactions added. Models in which some or all of the SM fields are allowed to propagate into the bulk have also been constructed [11]. It is found that stringent constraints are put upon the KK excitations of these models from current experiments. However, these limits are highly model dependent and it is difficult to draw general conclusions on the viability of the extra dimensional scenarios.

As discussed previously the brane world scenario has a natural setting in string theory; however, it can be studied from the effective field theory point of view. One difficulty of such an approach is that we are now dealing with field theories in higher dimensions which are known to have divergences when virtual loop effects are considered. Such radiative corrections are well tested in many precision measurements which have firmly established local quantum field theory as the correct framework for physics up to the weak scale. Most notable of these is the anomalous magnetic moments of the muon, $a_{\mu}$, and that of the electron, $a_{e}$. Currently the world average value for $a_{\mu}$ is $11659210(46) \times 10^{-10}$ and the difference between the theoretical and experimental value for $a_{\mu}$ is $\Delta a_{\mu} \equiv a_{\mu}^{e x p}-a_{\mu}^{S M}=$ $(43 \pm 45) \times 10^{-10}$. The E821 experiment at BNL plans to reduce the error to $0.35 \mathrm{ppm}$ [12]. This is clearly an important quantity for constraining any model of physics beyond the SM ; and is particularly so for the effective field theory approach to extra dimensions and brane world scenarios.

In this paper we study $a_{l}$ where $l=\mu, e$ in the both the ADD and the RS models with bulk neutrinos. We shall ignore the effects of neutrino mixings among the three families and concentrate only on the effects coming from extra dimensions and bulk neutrinos. A 
previous study 13 of $a_{\mu}$ in the ADD model used additional Higgs doublets and found that the dominant effect of bulk neutrinos also involved charged Higgs exchange. For the ADD case we shall use the minimal model where all the SM particles are localized on the brane and only a right-handed neutrino is allowed to propagate in the bulk [6]. We shall ignore the graviton and its KK excitations as this is discussed in 13 . For the RS scenario the two models with bulk neutrinos we use are given in [9] (brane Higgs) and [10] (bulk Higgs).

It is illustrative to begin our discussions with the gauge invariant contribution to $a_{l}$ from a massless neutrino in the SM. The Feynman diagrams are depicted in Fig. 1. and the mass insertion technique is employed for fermions. A simple calculation gives the result in standard notations:

$$
a_{l}^{\nu}=\frac{G_{F} m_{l}^{2}}{4 \pi^{2} \sqrt{2}}\left(\frac{5}{3}\right)
$$

where the superscript denotes the contribution from the active $\nu_{l}$ of the SM.

To see the effects of bulk neutrinos and extra dimensions on the anomalous magnetic moment we begin with the ADD case, with one extra dimension compactified into a circle of radius $\mathrm{R}$. Thus the geometry is $M^{4} \times T^{1}$. The brane where the SM particles reside is located at $z=0$, i.e. the origin of the extra dimension. The effective Lagrangian density in $4 \mathrm{D}$ involving the active neutrino and the bulk neutrinos, $N$, is given by

$$
\mathcal{L}=\int_{0}^{2 \pi R} d z \bar{N}\left(i \gamma^{\mu} \partial_{\mu}+i \Gamma_{5} \partial_{z}\right) N+y_{*} \int_{0}^{2 \pi R} d z \delta(z) \bar{L} H N_{R}+\text { h.c. }
$$

where $L$ is the left-handed SM lepton doublet, $H$ denotes the SM Higgs doublet. Our choice of the Clifford algebra representation, $\Gamma^{A}$, for $5 \mathrm{D}$ spacetime is : $\Gamma^{\mu}=\gamma^{\mu}$ for $\mu=0,1,2,3$ and $\Gamma^{5}=i \gamma^{5}$. The 5D Yukawa coupling, $y_{*}$, is dimensionful and is related to the dimensionless coupling $y$ via

$$
y_{*}=\frac{y}{M_{*}^{n / 2}}
$$

and $n=1$ for $5 \mathrm{D}$. We have neglected a possible higher dimensional bare Dirac mass term for simplicity. We implement the Kaluza-Klein ansatz by Fourier expanding the fields $N_{L, R}$ as follows:

$$
\begin{aligned}
& N_{R}=\frac{1}{\sqrt{2 \pi R}} \sum_{k=-\infty}^{\infty} n_{k R} e^{\frac{i k z}{R}}, \\
& N_{L}=\frac{1}{\sqrt{2 \pi R}} \sum_{k=-\infty}^{\infty} n_{k L} e^{\frac{i k z}{R}} .
\end{aligned}
$$


The mass terms necessary for our calculations are obtained by substituting the above into Eq.(5) and after electroweak breaking we have

$$
m_{D} \bar{\nu}_{l} n_{0 R}+m_{D} \sum_{k=1}^{\infty} \bar{\nu}_{l L}\left(n_{k R}+n_{-k R}\right)+\sum_{k=1}^{\infty} m_{k}\left(\bar{n}_{k L} n_{k R}-\bar{n}_{-k L} n_{-k R}\right)+\text { h.c. }
$$

where

$$
m_{k}=\frac{k}{R}, \quad m_{D}=\frac{y v}{\sqrt{4 \pi R M_{*}}}
$$

and $v=247 \mathrm{GeV}$. The contribution of the KK tower of bulk neutrino states to $a_{l}$ which is first order in $m_{D}$, comes from calculating the Feynman diagrams of Fig.2 where the crosses denote mass insertions via Eq.(9). It is given by

$$
a_{l}^{B N}=-\frac{g^{2}\left|m_{D}\right|^{2} m_{l}^{2}}{16 \pi^{2}} \sum_{k} \frac{1}{\left(w^{2}-m_{k}^{2}\right)^{2}}\left[\frac{2}{3}-\frac{m_{k}^{2}\left(w^{2}+5 m_{k}^{2}\right)}{6 w^{2}\left(w^{2}-m_{k}^{2}\right)}-\frac{m_{k}^{2}\left(w^{2}-2 m_{k}^{2}\right)}{\left(w^{2}-m_{k}^{2}\right)^{2}} \ln \frac{w^{2}}{m_{k}^{2}}\right]
$$

where $g$ is the $\mathrm{SU}(2)$ gauge coupling and $w$ is the mass of the $\mathrm{W}$-boson. As seen from Eq.(10) the KK states are separated by an equal amount of $1 / R$ and this spacing is small if the compactification radius is relatively large, such as micron size. The infinite sum in Eq.(11) can be approximated by an integral over the $m_{k}$. This integral is divergent and has to be cut off at the high mass region. A natural choice for this cutoff is $M_{*}$. When the leading term of the integrals is evaluated, the $M_{*}$ dependence is compensated by the corresponding scaling factor in $m_{D}$ such that the final result is finite in the limit $M_{*} \rightarrow \infty$. Explicitly, we have

$$
\begin{aligned}
a_{l}^{B N} & \cong-\frac{5 m_{l}^{2}|y|^{2}}{3(n-2) 2^{2 n+3} \pi^{\frac{n+4}{2}} \Gamma\left(\frac{n}{2}\right) M_{*}^{2}} & & n \neq 2, \\
& \cong-\frac{5 m_{l}^{2}|y|^{2}}{768 \pi^{3} M_{*}^{2}} \ln \frac{M_{*}^{2}}{w^{2}} & & n=2 .
\end{aligned}
$$

This contribution is negative and subtracts from the SM value except for $n=1$ where it adds. In these models the effect of the right-handed bulk neutrinos enters through mass insertions. An even number of such insertions are required due to the chiral nature of the SM interactions on the visible brane. This accounts for the factor of $|y|^{2}$. Higher order insertions will be subleading since $m_{D}$ is in general a small quantity. The overall negative sign in Eq.(12) is a result of the loop integration which led to Eq.(11). We show the result graphically in Fig. 3, along with the experimental uncertainty on $a_{\mu}$. The Yukawa coupling is at $y=1$ as an example. The contribution from two extra dimensions is nearly as large as for one, due to the logarithmic dependence in Eq.(12). However, the contribution is still well below what is measurable currently and in the near future.

The anomalous moment of the electron is smaller due to the two powers of lepton mass in Eq. (12). The contribution to $a_{e}$ from the bulk neutrinos is at largest around $10^{-15}$, whereas the experimental error is at around $10^{-11}$. 
The physics of bulk neutrinos in the RS model is different from that of the ADD case. We study both of these cases to examine whether the contribution can be detected in a precision measurement of $a_{l}$. In the context of the RS scenario, only 5D models have been studied in any detail. Below we outline the essential steps for obtaining the couplings of bulk neutrinos with the brane fermions which are necessary for our calculations.

The action for the bulk neutrino in the RS model [see Eq.(2)] is given by [9]

$$
S=\int d^{4} x \int d \phi \sqrt{G}\left[E_{a}^{A} \frac{i}{2} \bar{\Psi} \gamma^{a}\left(\vec{\partial}_{A}-\overleftarrow{\partial}_{A}\right) \Psi-m \operatorname{sgn}(\phi) \bar{\Psi} \Psi\right]
$$

where $\gamma^{a}=\left(\gamma^{\mu}, i \gamma^{5}\right), G=\operatorname{det}\left(G_{A B}\right), E_{a}^{A}=\operatorname{diag}\left(e^{\sigma}, e^{\sigma}, e^{\sigma}, e^{\sigma}, \frac{1}{r_{c}}\right)$ is the inverse vielbein, $\sigma=k r_{c}|\phi|$, and $m$ is a Dirac mass. We have neglected the spin connection term which plays no role in our investigation. The bulk neutrinos are Dirac fermions and the left and right-handed projections come from $\Psi_{L, R} \equiv \frac{1}{2}\left(1 \mp \gamma^{5}\right)$ with the periodic boundary condition $\Psi_{L, R}(x, \pi)=\Psi_{L, R}(x,-\pi)$. The KK decomposition of the $\Psi$ is given by

$$
\Psi_{L, R}(x, \phi)=\sum_{n} \frac{e^{2 \sigma}}{\sqrt{r_{c}}} \psi_{L, R}^{n}(x) \hat{f}_{L, R}^{n}(\phi)
$$

and gives rise to the set of $4 \mathrm{D}$ Dirac equations

$$
S=\sum_{n} \int d^{4} x\left(\bar{\psi}_{L, R}^{n} i \gamma^{\mu} \partial_{\mu} \psi_{L, R}^{n}-m_{n} \bar{\psi}_{R, L}^{n} \psi_{L, R}^{n}+\text { h.c. }\right)
$$

with the conditions

$$
\int_{0}^{\pi} d \phi e^{\sigma} \hat{f}_{L}^{m *} \hat{f}_{L}^{n}=\int_{0}^{\pi} d \phi e^{\sigma} \hat{f}_{R}^{m *} \hat{f}_{R}^{n}=\delta^{m n}
$$

and

$$
\left( \pm \frac{1}{r_{c}} \partial_{\phi}-m\right) \hat{f}_{L, R}^{n}+m_{n} e^{\sigma} \hat{f}_{R, L}^{n}=0 .
$$

For convenience we define the variables $\epsilon \equiv e^{-k r_{c} \pi}, x_{n} \equiv m_{n} /(k \epsilon), t \equiv \epsilon e^{\sigma}$ and the rescaled function $\hat{f}_{n}^{L, R}(\phi) \equiv \sqrt{k r_{c} \epsilon} f_{n}^{R, L}(t)$. We are interested in the mass of the KK modes, $m_{n}$. These can be found by way of the solutions for $f_{R}$. The eigenvalues $m_{n}$ are determined by the roots of Bessel functions of order $\nu \equiv m / k$ given below :

$$
J_{\nu-\frac{1}{2}}\left(x_{n}\right)=0
$$

We also note that the value of $f_{n}^{R}(1)=\sqrt{2}$ for all $n \neq 0$ at the visible brane can be obtained from the above equations and the appropriate boundary conditions which satisfy the orbifold symmetry.

In order to see the implications of Eq. (19) we must know $\nu$ and also $k$ and $r_{c}$. If the bulk neutrino partners with the active $\nu_{L}$ to form a light neutrino then $\nu \simeq 1.1-1.5$ [10]. The KK bulk neutrino states are approximately equally spaced as the roots of Eq.(19). 
They are given approximately by $n \pi$ for $\nu \approx 1$. Since the solution of the hierarchy problem requires $k r_{c} \approx 12$ and also $k \lesssim M_{5}$, then the K.K. states will be of order the weak scale and the bulk Dirac mass $m \sim M_{5}$. This is independent of whether the Higgs doublet resides only on the visible brane or in the bulk.

To proceed we introduce the Higgs doublet which couples the bulk field to the chiral lepton doublet which is confined on the brane. We first examine the case which has this scalar field, $h_{0}(x)$, also localized on the visible brane. The relevant action terms are

$$
S=\int d^{4} x \epsilon^{4}\left[e^{2 k r_{c} \pi} \partial^{\mu} h_{0}^{\dagger} \partial_{\mu} h_{0}-\lambda\left(\left|h_{0}\right|^{2}-\frac{v_{0}^{2}}{2}\right)^{2}-\left(\frac{Y_{5}}{\sqrt{M_{5}}} \bar{L}_{0}(x) h_{0}(x) \Psi_{R}(x, \pi)+h . c .\right)\right]
$$

where the subscript 0 denotes bare fields and $Y_{5}$ is the dimensionless Yukawa coupling. As noted in [4], in order to get the canonical normalization of fields on the brane one must do the rescalings: $h_{0} \rightarrow h e^{k r_{c} \pi}$ and $L_{0} \rightarrow e^{\frac{3}{2} k r_{c} \pi} L$ and obtain $v=v_{0} \epsilon$ which we identify as the weak scale. After electroweak symmetry breaking, we can use Eqs. (15, 20) and the rescaling relations, to find the coupling between $\nu_{l L}$ and the $n^{\text {th }} \mathrm{KK}$ bulk neutrino:

$$
\begin{aligned}
y_{n} v & =Y_{5} v \sqrt{\frac{k}{2 M_{5}}} f_{n}^{R}(1) \\
& =Y_{5} v \sqrt{\frac{k}{M_{5}}}
\end{aligned}
$$

for $n \neq 0$. The zero mode coupling is very small and is interpreted as the light neutrino mass. On the other hand, the mass of the KK excitations are of the weak scale. Taking $\nu \simeq 1.1$ and $k \sim 10^{18} \mathrm{GeV}$ as an example, we obtain $m_{1} \sim 133 \mathrm{GeV}$ and $m_{2} \sim 266$ $\mathrm{GeV}$ etc. Thus we can expect most KK modes to be heavier than the $\mathrm{W}$ boson. The contribution to $a_{l}$ from the bulk neutrinos in the RS model with brane Higgs can now be obtained from Eq.(11). Keeping only the leading term we find

$$
\begin{aligned}
a_{l}^{R S} & \simeq \frac{\left|Y_{5}\right|^{2} m_{l}^{2} k}{4 \pi^{2} M_{5}}\left(-\frac{5}{6}\right) \sum_{n=1}^{\infty} \frac{1}{m_{n}^{2}} \\
& \simeq-\frac{5\left|Y_{5}\right|^{2} m_{l}^{2}}{24 \pi^{4} k M_{5} \epsilon^{2}} \sum_{n=1}^{\infty} \frac{1}{n^{2}} \quad(\text { for } \nu=1.1) \\
& =-\frac{5\left|Y_{5}\right|^{2} m_{l}^{2}}{144 \pi^{2} k M_{5} \epsilon^{2}},
\end{aligned}
$$

where we have taken the sum to infinitely many KK states. The masses of the KK modes are given by the roots of the Bessel function. We approximate the roots as being spaced by $\pi n$. This approximation is best for the lower values of $\nu$. For example, for $\nu \approx 1.5$ they are spaced as $(n+5 / 4) \pi$, so for this case the result is slightly smaller than in the expression above. Figure 4 shows the magnitude of the correction to $a_{\mu}$ from the bulk 
neutrinos in the RS model. For the illustrative purposes of this figure, we have taken $k \epsilon \sim v$ and also $M_{5} \epsilon \sim v$. Again, the contribution to $a_{e}$ is several orders of magnitude below current experimental uncertainty due to the $m_{e}^{2} /\left(k M_{5} \epsilon^{2}\right)$ suppression.

It was pointed out in [10] that the phenomenology of the SM on the visible brane depends on whether the Higgs field is allowed to propagate in the bulk or not. Here we explore the consequences of bulk Higgs on $a_{l}$. The action of the bare bulk Higgs field, $H_{0}$ we are interested in is given by

$$
\begin{aligned}
S_{H}= & \int d^{4} x \int_{-\pi}^{\pi} d \phi \sqrt{G}\left[G^{A B} D_{A} H_{0}^{\dagger} D_{B} H_{0}-\frac{\lambda_{B}}{4 M_{5}}\left(H_{0}^{\dagger} H_{0}-\frac{v_{0}^{3}}{2}\right)^{2}\right] \\
& -\left[\frac{Y_{B}}{M_{5}} \int d^{4} x \epsilon^{4} \bar{L}_{0} H_{0}(x, \pi) \Psi_{R}(x, \pi)+\text { h.c. }\right]
\end{aligned}
$$

where the Yukawa coupling $Y_{B}$ is dimensionless, and $D_{A}$ is the gauge covariant derivative. Spontaneous symmetry breaking is achieved by $H_{0} \rightarrow\left(H+v_{0}^{\frac{3}{2}}\right) / \sqrt{2}$. The weak scale is obtained from the gauge terms (see [10]) and is

$$
v=\epsilon v_{0} \sqrt{r_{c} v_{0}}
$$

The mass insertion can be obtained from the Yukawa term of Eq.(25), Eq.(15), and the field rescaling. Explicitly the $\nu_{L} \psi_{n}$ coupling is given by

$$
\frac{1}{\sqrt{2}} Y_{B} v_{0} \sqrt{v_{0} k} \frac{\epsilon}{M_{5}} f_{n}^{R}(1)=Y_{B} v \sqrt{\frac{k}{M_{5}}}\left(\frac{1}{\sqrt{M_{5} r_{c}}}\right)
$$

for all KK bulk neutrinos. This has a suppression factor of $1 / \sqrt{M_{5} r_{c}}$, as compared with the brane Higgs case. This suppression factor occurs in all bulk Higgs Yukawa couplings, and is not unique to the coupling of the bulk neutrinos. For example it also appears in brane fermion mass terms, such as the electron mass. This factor is interpreted as a general rescaling of the Yukawa coupling. Therefore the expression for $a_{l}$ is the same as in the brane Higgs case except for the rescaling of the coupling.

We have examined the effect of virtual bulk neutrinos by calculating the correction to the anomalous magnetic moment of the charged leptons. We have employed three different models: a factorizable geometry with up to six extra dimensions (ADD), and two versions of the Randall-Sundrum scenario with one extra dimension. In each case we take the simplest model, with only the bulk neutrino and/or the Higgs field propagating in the bulk. This demonstrates the key features of the extra dimensional neutrino scenarios. A Kaluza-Klein tower of bulk neutrinos must be summed in order to calculate the correction. In all cases the correction is finite and does not diverge as the higher dimensional scale increases, which corresponds to taking the KK tower to be infinite. This indicates that the predictions are robust. It is interesting to note how the new physics is probed by 
comparing the Eqs. (田, 12), and (23). In the SM the size of $a_{l}$ is set by the weak scale whereas in the ADD case this is replaced by the scale $M_{*}$. On the other hand in the RS model the determining scale is the redshifted scale $M_{5} \epsilon$. In addition, the contribution to the electron magnetic moment is significantly smaller than that of the muon, because of suppression factor, $\left(m_{l} / M_{*}\right)^{2}$ or $\left(m_{l} / \epsilon k\right)^{2}$. In all scenarios, the contribution from the bulk neutrino modes is currently below experimental precision. However the RandallSundrum models are close to being probed by the next generation of g-2 measurements for the muon.

While this paper was being written a preprint appeared that investigated $a_{l}$ in the RS scenario with the SM chiral fermions and gauge bosons all allowed to propagate in the bulk [14].

This work is partially supported by the Natural Science and Engineering Research Council of Canada 


\section{References}

[1] N. Arkani-Hamed, S. Dimopoulos, and G. Dvali, Phys. Lett. B429, (1998) 263, and Phys. Rev. D59,(1999) 086004

[2] I. Antoniadis, N. Arkani-Hamed, S. Dimopoulos, and G. Dvali,Phys. Lett. B436, (1998) 257

[3] J. Polchinski, hepph/9611050

[4] L. Randall and R. Sundrum, Phys. Rev. Lett. 83, (1999) 3370

[5] N.Arkani-Hamed, S.Dimopoulos, G.Dvali, and J. March-Russell, hepph/9811448

K.R. Dienes, E. Dudas, and T. Gherghetta, Nucl. Phys. B557, (1999) 25

[6] G. Dvali and Yu. Smirnov, Nucl. Phys. B563, (1999) 63

[7] R.Babieri, P. Creminelli, and A. Strumia, hepph/0002199

R.N. Mohapatra and A. Pérez-Lorenzana, hepph/0006278

[8] A.Fraggi and M. Pospelov, Phys. Lett. B458, (1999) 237

A.Das and O.Kong, Phys. Lett. B470, (1999) 149

G.McLaughlin and J.N. Ng, Phys. Lett. B470, (1999) 157 and hepph/0003023

R.N. Mohapatra and A. Pérez-Lorenzana, Nucl. Phys. B576, (200) 466

[9] Y. Grossman and M. Neubert, Phys. Lett. B474, (2000) 361

[10] C.H.V. Chang and J.N. Ng, hepph/9906164

[11] H. Davoudiasl, J.L. Hewett, and T. Rizzo, Phys. Rev. Lett. 84, (2000) 2080 and Phys. Lett. B473, (2000) 43

A. Pomeral, hepph/9911294

[12] For a review see B.L. Roberts, hepex/0002005 and R.Carey, talk at ICHEP 2000, July 2000 Osaka, Japan for the most recent result.

[13] M.L. Graesser, Phys. Rev. D61,(2000) 074019

[14] H. Davoudiasl, J.L. Hewett, and T.G. Rizzo hepph/0006097 v3 
Figure 1: Standard Model contribution to g-2 of the electron in the unitary gauge, which has a neutrino as an intermediate state. The cross represents a mass insertion, which can be on either external line.

Figure 2: First order bulk neutrino contribution to g-2 of the electron in the unitary gauge. As in Fig. 1 there is an additional diagram with the electron mass insertion on the other external line.

Figure 3: Shows the predicted contribution to g-2 for the muon from the bulk neutrinos for different numbers of extra dimensions in the ADD scenario. The horizontal dashed lines show the current (upper) and future expected (lower) experimental sensitivities. The solid line shows the contribution for one extra dimension, while the dot-dashed line shows the contribution for two extra dimensions. The lowest dashed line on the plots shows the contribution for three extra dimensions.

Figure 4: Shows the predicted contribution to g-2 from the bulk neutrinos for the RandallSundrum scenario. The dashed horizontal lines show experimental sensitivity as in Fig 3. The solid line shows the contribution from bulk neutrinos when the Higgs boson is restricted to the $3+1$ dimensional brane. 


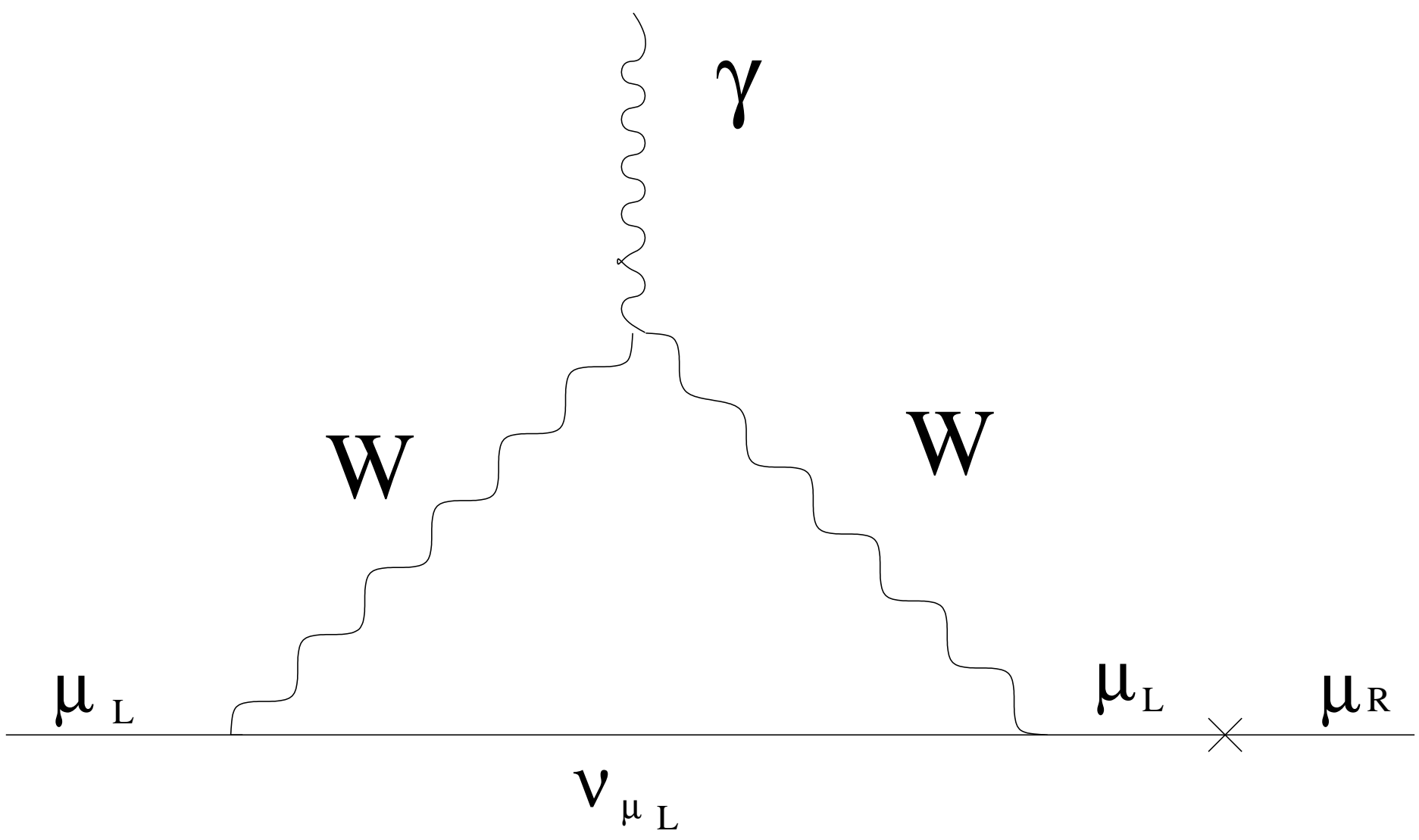




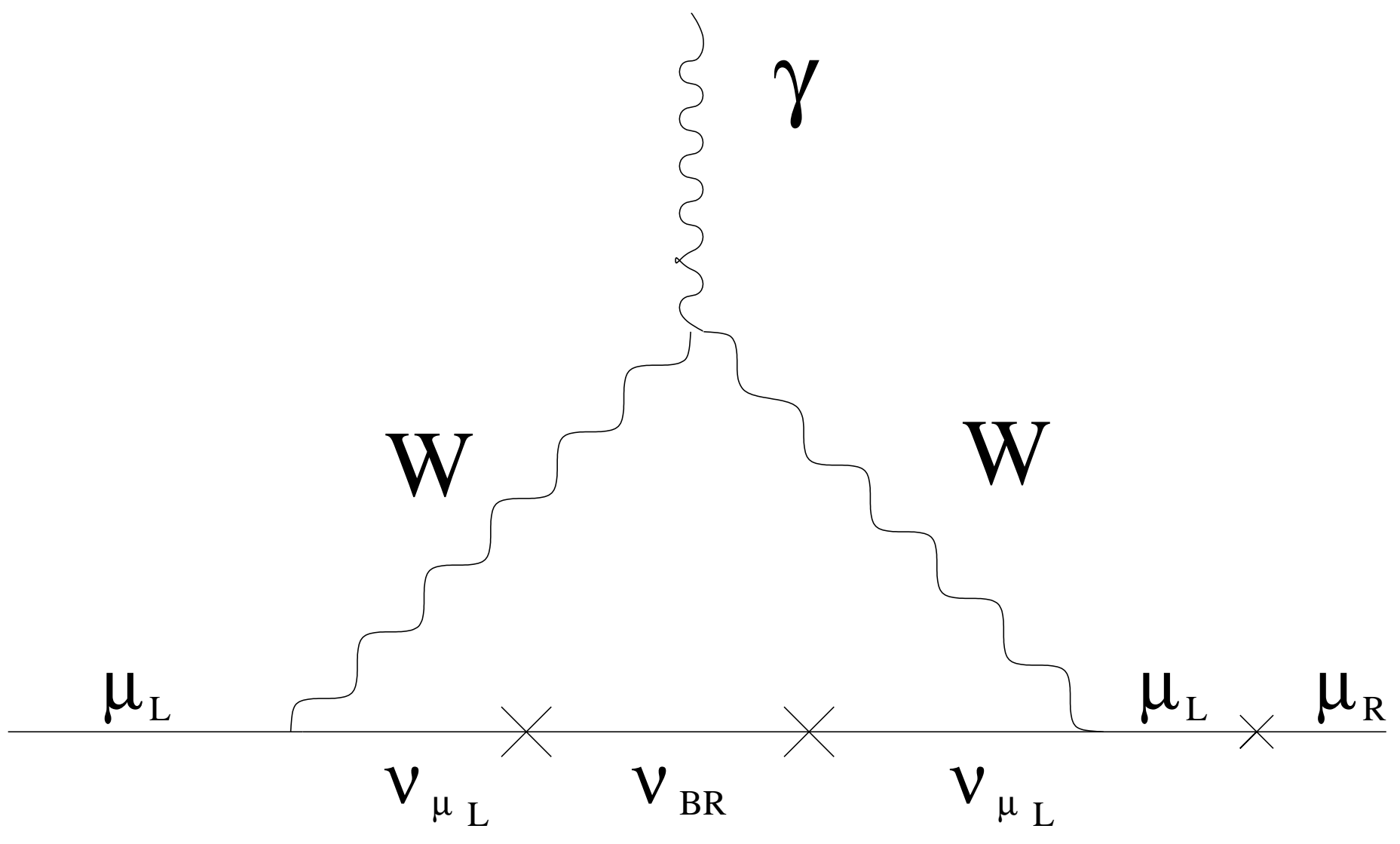




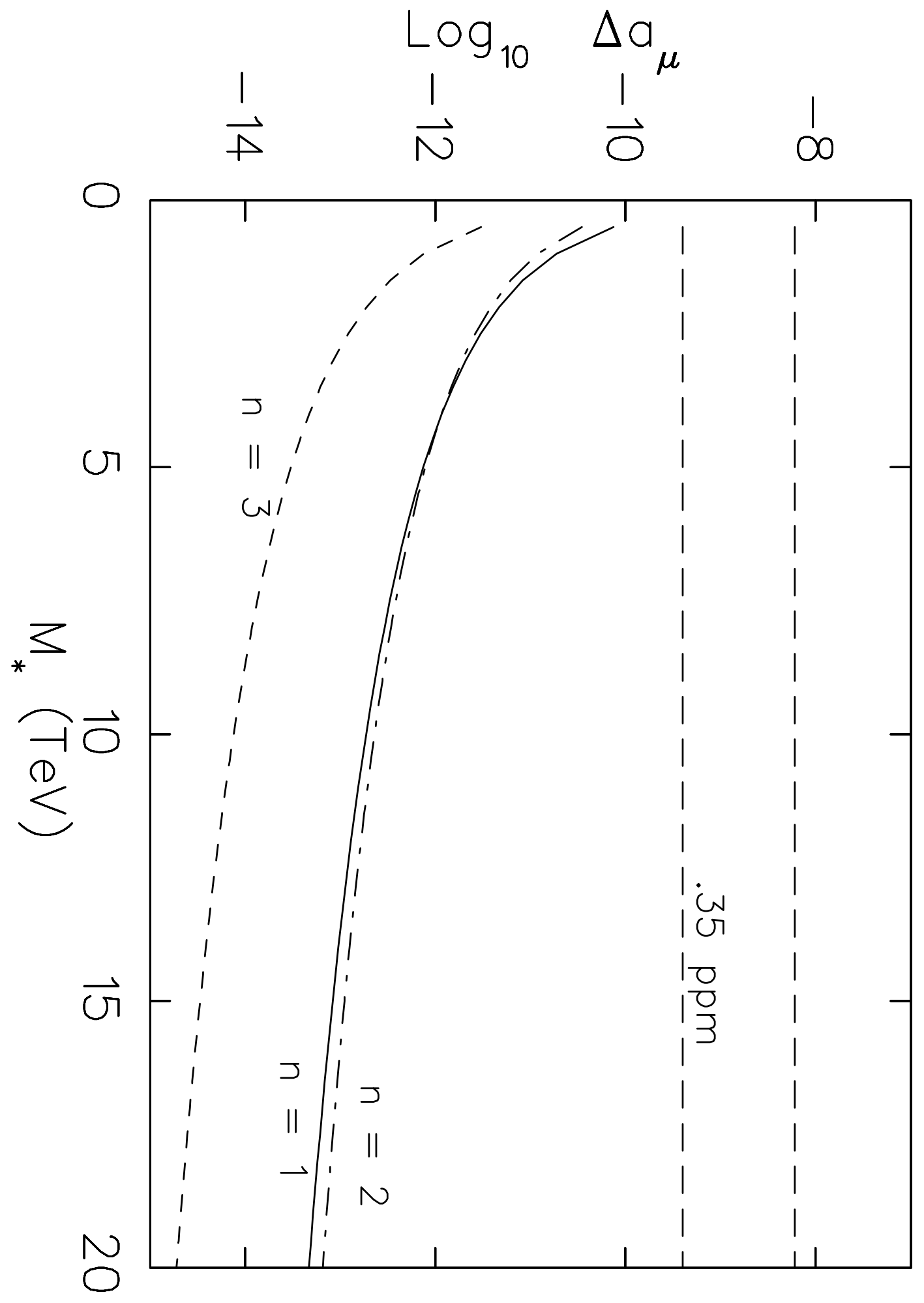




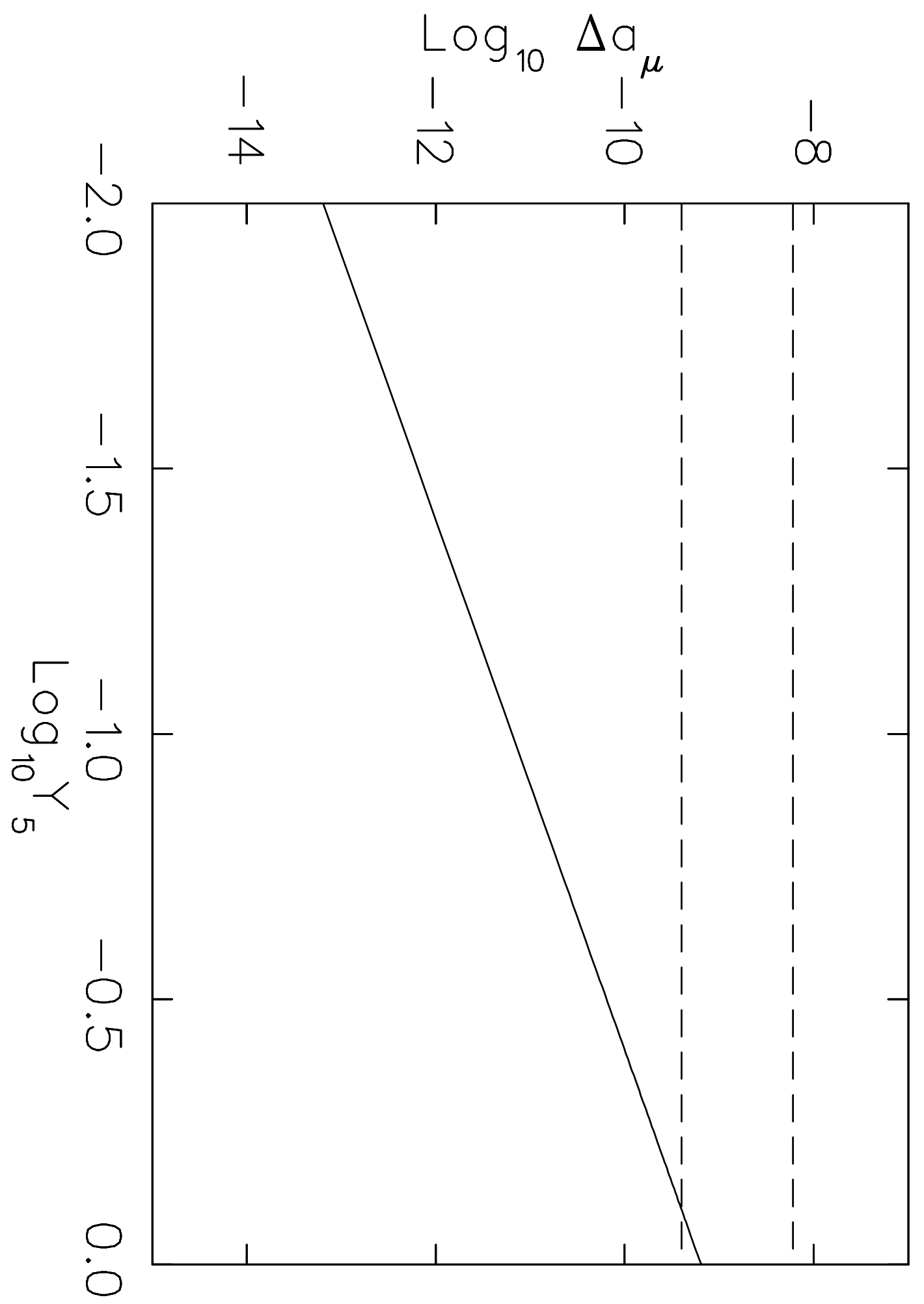

